\title{
Molecular Enginecring. Part 12. Hemicarcerand Having a Metal Coordinating Ligand at a Hetero-Bridge
}

\author{
Byounghei Ye and Kyungsoo Paek" \\ CAMDRC and Deparment of Chemistry, Soongsil University, Seoul 156-743, Korea. "E-mail kpaek(d)sstiac:kv. \\ Received October 7, 2005
}

Key Words : Iemicarcerand, Itemicarceplex, Metal coordination, Container dimer

Resorcin|4/arene-based container hosts such as carcerand, ${ }^{1}$ hemicarcerand, ${ }^{2}$ velerand, ${ }^{3}$ polyvelcraplex, ${ }^{4}$ and sellassembled molecular capsule have been characterized with the potential applications as molecular reactors, selective storage, delivery and controlled-releasing systems. Recently, the research ficlds of supramolecular systems are being expanded from single host-guest systems to high ordered macromolecular systems such as polymeric nanostructure or seli-assembly monolayer. ${ }^{7}$

Dimeric container system could duplex the function of monomeric container molecule and a well-controlled manipulation of dimeric container system would result in a new highly accumulated information storage system. By Cram group, various helerobridged hemicarcerplexes were synthesized from diol $\mathbf{1}$, in which the fourth bridging unit differs from the other three bridging units. ${ }^{x}$ And a tourth bridging unit has been used to connect with another hemicarcerand to give various dimeric hemicarceplexes."

Metal coordination to promote the self-assembly of highordered and well-defined supramolecular architectures has become an important synthetic strategy because it allows well defined geometry, coordination number, and a range of binding strengths." IJere we report on the synthesis and binding properties of hemicarcerand 2 which has a metal coordinating cyanophenyl unit and its preliminary characteristics as a dimeric self-assembly by metal coordination.

Diol $1^{\text {tc }}$ was synthesized in $44 \%$ yield from tetrol ${ }^{11}$ using Sheman's templating procedure ${ }^{12}$ (NMP, $\mathrm{Cs}_{2} \mathrm{CO}_{3}, 3$ equiv of $\left.\mathrm{TsO}\left(\mathrm{CH}_{2}\right)_{4} \mathrm{OTs}, 25^{\circ} \mathrm{C}, 12 \mathrm{~h}\right)$. Under the dilution condition. diol 1 was reacted with 3,5 -bis(bromomethyl)-benzonitrile ${ }^{13}$ in a mixlure of $\mathrm{C}_{2} \mathrm{CO}_{3}$ and NMP at $60^{\circ} \mathrm{C}$ to afford hemicarcerand $2(\omega \mathrm{CHCl}$ in $38 \%$ yicld after chromatographic purification (hexane : $\mathrm{CHCl}_{3}=2: 1$ ) and secrystallization $\left(\mathrm{CH}_{3} \mathrm{OH}\right)$. The initial product 2(a) NMP seems to be changed to $2\left(a \mathrm{CHCl}_{3}\right.$ by mass-driven exchange. Heterobridged hemicarecrand $2\left(\alpha \mathrm{CHCl}_{3}\right.$ was characterized by 'H NMR, FT-IR and FAB+ Mass spectra. Hemicarceplexes 2 a-g were obtained by heating a mixture of $2(a) \mathrm{CHCl}_{3}$ and an excess of guests. To a round bottom llask equipped with an argon gas inlet were added hemicarecrand $2\left(a \mathrm{CHCl}_{3}\right.$ and various guests as solvents (>1000 fold). The mixture was heated at 130-160 ${ }^{\circ} \mathrm{C}$ for 36-64 hours. The cooled reaction mixtures were flooded with $\mathrm{CH}_{3} \mathrm{OH}$ and the precipitates were filtered and dried in vacho.

Table 1 shows the conditions and results for thermally indueed complexation, and chemical shifts of 'H VMR spectra for complexed guest in CDCl: at $25^{\circ} \mathrm{C}$. The sizes and shapes of guest candidates must be complementary enough to the host's portals and interiors so that constrictive and intrinsic binding taken together allow isolable and manipulable hemicarceplexes. The chemical shif changes of guest protons illustrate the relative orientation of guest in the interior of hemicarcerand. Due to the shielding effect of aromatic units, protons close to aromatic $\pi$-cloud shift to upfield. In general the upfield-shili of protons on meta- or para-substituent is larger than that of ortho-substituent. Trisubstitued benzenes enter slowly than disubstitued ones do. From the complexation ratio of disubstitued benzenes the complexation elfieiency decreases in the order of para- $>$ meta- >> ortho-disubstituted bencene. Interestingly methoxy group favors complextion than methyl group does.

Table 2 shows the hall-lives for decomplexation. It is assumed that decomplexation exhibits the lirst order behavior in large excess of solvent over several half-lives. Decomplexation half-life for hemicarceplex $\mathbf{2 a}$ is about 2.5 times longer than that for hemicarceplex $\mathbf{2 b}$ whose hall-lile is about 17 times longer than that of hemicarceplex $2 \mathrm{e}$. Clearly the steric hindrance of guest imposes a large activation energy barrier to escape the portal of host.

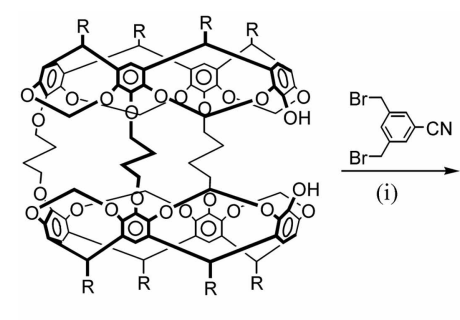

1
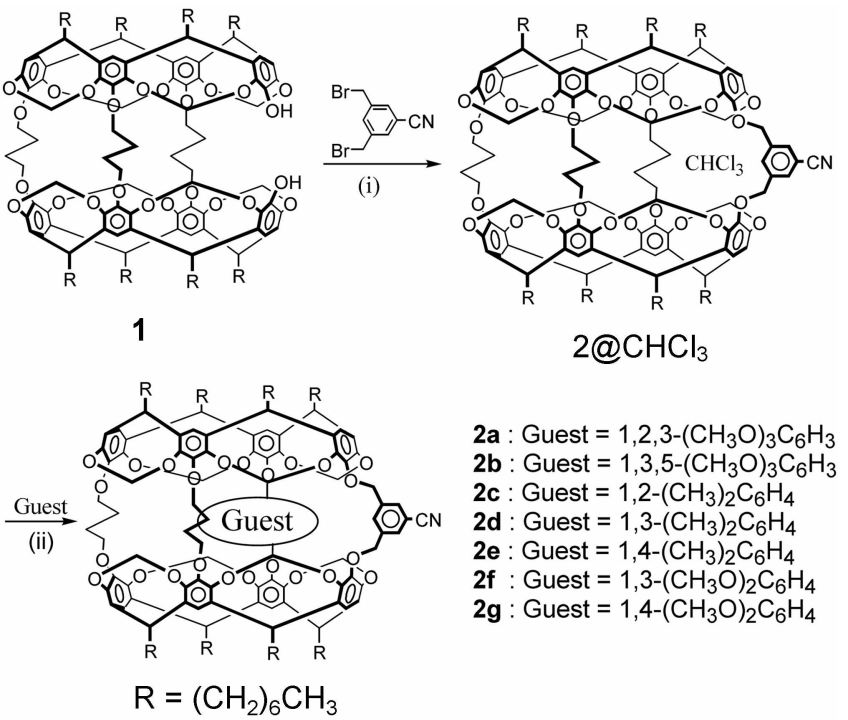

$2 @ \mathrm{CHCl}_{3}$

2a : Guest $=1,2,3-\left(\mathrm{CH}_{3} \mathrm{O}\right)_{3} \mathrm{C}_{6} \mathrm{H}_{3}$ 2b: Guest $=1,3,5-\left(\mathrm{CH}_{3} \mathrm{O}\right)_{3} \mathrm{C}_{6} \mathrm{H}_{3}$ 2c : Guest $=1,2-\left(\mathrm{CH}_{3}\right)_{2} \mathrm{C}_{6} \mathrm{H}_{4}$ 2d: Guest $=1,3-\left(\mathrm{CH}_{3}\right)_{2} \mathrm{C}_{6} \mathrm{H}_{4}$ 2e : Guest $=1,4-\left(\mathrm{CH}_{3}\right)_{2} \mathrm{C}_{6} \mathrm{H}_{4}$ 2f: : Guest $=1,3-\left(\mathrm{CH}_{3} \mathrm{O}\right)_{2} \mathrm{C}_{6} \mathrm{H}_{4}$ $2 \mathrm{~g}:$ Guest $=1,4-\left(\mathrm{CH}_{3} \mathrm{O}\right)_{2} \mathrm{C}_{6} \mathrm{H}_{4}$

Scheme 1. Conditions: (i) Cs.CONMP, $60^{\circ} \mathrm{C}, 38 \%$, (ii) $130-160$ "C., 3 days. 
Table 1. Conditions and results for complexation and chemical shifts of complexed guest proton $\left(400 \mathrm{MHz}{ }^{1} \mathrm{H} \mathrm{NMR}, \mathrm{CDCl}_{3}, 25{ }^{\prime \prime} \mathrm{C}\right)$

\begin{tabular}{|c|c|c|c|c|c|c|c|c|}
\hline No & guest structure & $T\left({ }^{\circ} \mathrm{C}\right)$ & $\begin{array}{c}t \\
\text { (hours) }\end{array}$ & $\begin{array}{l}\text { Isolation } \\
\text { yield (\%) }\end{array}$ & \multicolumn{2}{|c|}{$\begin{array}{c}\delta(\mathrm{ppm}) \text { of } \\
\text { complexed guest }\end{array}$} & 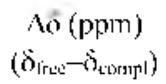 & $\begin{array}{c}\text { complexation } \\
\text { ratio }(\%)\end{array}$ \\
\hline $2 \mathbf{a}$ & & 160 & 36 & 86 & $\begin{array}{l}\mathrm{I}_{\mathrm{a}} \\
\mathrm{H}_{\mathrm{b}} \\
\mathrm{H}_{\mathrm{c}} \\
\mathrm{H}_{\mathrm{a}}\end{array}$ & $\begin{array}{r}-0.56 \\
-0.46 \\
6.58 \\
6.60\end{array}$ & $\begin{array}{r}4.41 \\
4.32 \\
0 \\
0.39\end{array}$ & $46 \%$ \\
\hline $2 b$ & & 160 & 36 & 66 & $\begin{array}{l}\mathrm{H}_{\mathrm{a}} \\
\mathrm{H}_{\mathrm{b}},\end{array}$ & $\begin{array}{c}-0.66 \\
a\end{array}$ & $\begin{array}{c}4.50 \\
\mathrm{a}\end{array}$ & $52 \%$ \\
\hline $2 c$ & & 130 & 64 & 95 & $\begin{array}{l}\mathrm{IL} \\
\mathrm{IJ} \\
\mathrm{IJ}_{\mathrm{L}}\end{array}$ & $\begin{array}{c}-1,74 \\
a \\
a\end{array}$ & $\begin{array}{c}4,11 \\
a \\
a\end{array}$ & $44 \%$ \\
\hline $2 d$ & $H^{c}$ & 130 & 64 & 95 & $\begin{array}{l}\mathrm{H}_{\mathrm{c}} \\
\mathrm{H}_{\mathrm{b}} \\
\mathrm{H}_{\mathrm{c}} \\
\mathrm{IJ}_{\mathrm{l}}\end{array}$ & $\begin{array}{r}-2.00 \\
5.92 \\
5.92 \\
5.92\end{array}$ & $\begin{array}{l}432 \\
093 \\
1.07 \\
1.21\end{array}$ & $59 \%$ \\
\hline $2 \mathrm{e}$ & & 130 & 64 & 95 & $\begin{array}{l}\mathrm{II}_{\mathrm{a}} \\
\mathrm{I} \mathrm{I}_{\mathrm{b}}\end{array}$ & $\begin{array}{r}-1.99 \\
5.92\end{array}$ & $\begin{array}{l}431 \\
115\end{array}$ & $66 \%$ \\
\hline $2 \mathrm{f}$ & $H^{d}$ & 130 & 64 & 75 & $\begin{array}{l}\mathrm{Il}_{\mathrm{il}} \\
\mathrm{H}_{\mathrm{i}} \\
\mathrm{IL}_{\mathrm{c}} \\
\mathrm{H}_{\mathrm{i}}\end{array}$ & $\begin{array}{r}-0.60 \\
4.80 \\
4.80 \\
4.80\end{array}$ & $\begin{array}{l}4.40 \\
1.70 \\
1.74 \\
2.40\end{array}$ & $64 \%$ \\
\hline $2 g$ & & 130 & 64 & 65 & $\begin{array}{l}\mathrm{H}_{\text {il }} \\
\mathrm{H}_{\mathrm{H}}\end{array}$ & $\begin{array}{r}-0.46 \\
5.30\end{array}$ & $\begin{array}{l}4.23 \\
1.35\end{array}$ & $74 \%$ \\
\hline
\end{tabular}

Signal obscured by other peaks.

Table 2. Half-lives for decomplexation of $2 \cdot$ Guest in $\mathrm{Cl}_{2} \mathrm{CDCDCl}_{2}$ at $80^{\circ} \mathrm{C}$

\begin{tabular}{ccc}
\hline complex No. & (juest & $\mathrm{t}_{1: 2}(h)$ \\
\hline 2a & $1,2,3-\left(\mathrm{CH}_{3} \mathrm{O}_{3} \mathrm{C}_{6} \mathrm{H}_{3}\right.$ & 167 \\
2b & $1,3,5-\left(\mathrm{CH}_{3} \mathrm{O}_{3} \mathrm{C}_{6} \mathrm{H}_{3}\right.$ & 69 \\
$\mathbf{2 e}$ & $1,4-\left(\mathrm{CH}_{3}\right)_{2} \mathrm{C}_{6}, \mathrm{H}_{4}$ & 4 \\
\hline
\end{tabular}

As shown in Scheme 2 metal coordinated dimeric container molecular system was attempted with hemicarceplex 2(d) $\mathrm{CHCl}_{3}$ and $\mathrm{Pd}(\mathrm{PhCN})_{2} \mathrm{Cl}_{2}$ or $\mathrm{Pt}(\mathrm{dppp}) \mathrm{OTf}_{3}$. Figure 1 shows the partial FT-IR spectra of hemicarceplex $2 \Leftrightarrow \mathrm{CHCl}_{3}$ and their metal complexes. The stretching band of cyano group at $2231 \mathrm{~cm}^{-1}$ for hemicarcerand 2 was not changed significantly at $2: 1$ molar ration of $2: \mathrm{Pd}(\mathrm{PhCN})_{2} \mathrm{Cl}_{2}$. But at $2: 1$ molar ratio of $2: \mathrm{Pt}(\mathrm{dppp})_{2} \mathrm{OT}_{22}$, new stretching band of cyano group at $2250 \mathrm{~cm}^{-1}$ was appeared with that of free host 2 at $2231 \mathrm{~cm}^{-1}$.

But any chemical shift change of hemicarcerand 2 upon complexation by ${ }^{1} \mathrm{H}$ or ${ }^{13} \mathrm{C}$. NMR spectra couldn't be observed presumably due to the weak metal coordination strength of eyano group. The life time of metal coordinated complex seems too shorter than NMR time scale to be detected. The partial evidence of metal coordination by IR spectrum was possible due to the shorter IR time scale. It is desirable to adopt stronger ligand such as pyridyl to get stable metal- 

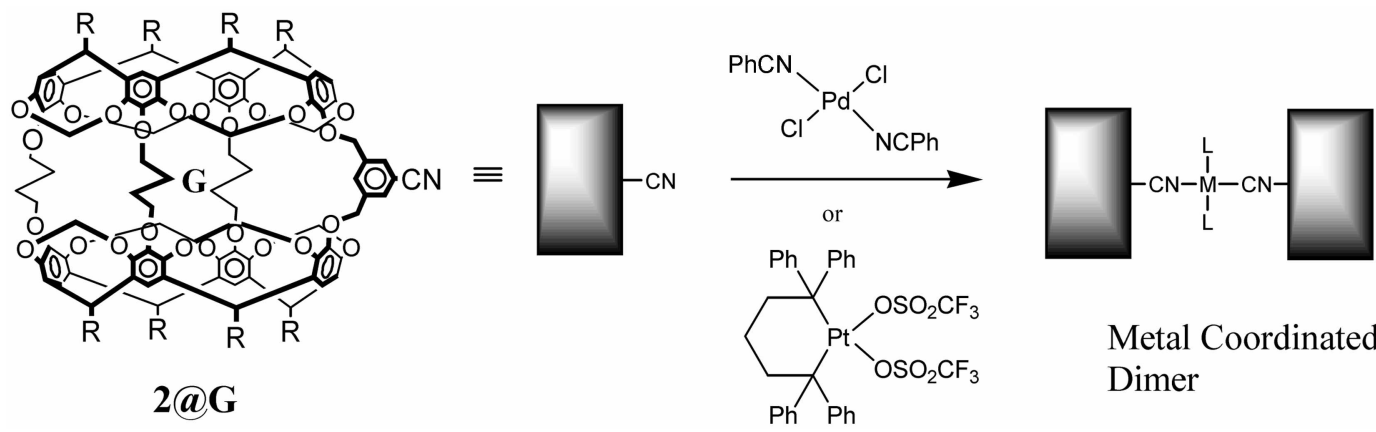

Metal Coordinated Dimer

Scheme 2. Proposed formation of metal coordinated dimeric container molecular system.

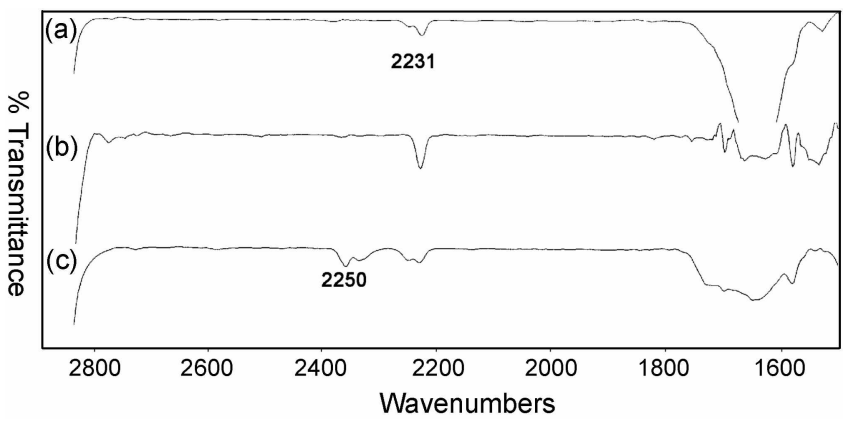

Figure 1. FT-[R spectra of (a) Hemicarceplex $2\left(a \mathrm{CHCl}_{7}\right.$, (b) $2\left(a \mathrm{CHCl}_{3} \cdot \mathrm{Pd}(\mathrm{NCPh})_{2} \mathrm{Cl}_{3}\right.$, (c) $2\left(0 \mathrm{CH} \mathrm{Cl}_{3} \cdot \mathrm{Pt}(\mathrm{dppp}) \mathrm{OTT}_{3}\right.$

coordinated dimeric container suprastruclure. When container molecular systems with multi-ligands were developed, highly organized 2-D net-work of container molecular systems could be constructed.

\section{Experimental Section}

3,5-Bis(bromomethyl)-benzonitrile. A mixture of 3,5dimethylbenzonitrile (1.0 g, $7.6 \mathrm{mmol})$, NBS (1.35 g, 3.8 mol) and catalytic amount of AJBN in $50 \mathrm{~mL}$ of dry $\mathrm{CH}_{2} \mathrm{Cl}_{2}$ was stirred under visible light and argon atmosphere for 1 hr at $25^{\circ} \mathrm{C}$. To the mixture were added more NBS (1.35 g, 3.8 mmol) and catalytic amount of AIBN. After 1 day, the solvent was evaporated under vacuum. The residue was dissolved in $\mathrm{CH}_{2} \mathrm{Cl}_{2}$, wash with water and $\mathrm{C}_{2} \mathrm{H}_{2} \mathrm{Cl}_{2}$ layer was dried over $\mathrm{MgSO}_{4}$, and purified by silica gel chromatography with a mixture of $\mathrm{C}_{2} \mathrm{H}_{2} / \mathrm{Hexane}_{(6: 1)}$ as a mobile phase to give $703 \mathrm{mg}(32 \%)$ of product: FT-IR (KBr) 2230 $\mathrm{cm}^{-1}$ (1 $\left.\mathrm{cN}^{\mathrm{N}}\right)$; 'H N.MR (400 MHz, CDCl $) \delta 4.45(\mathrm{~s}, 6 \mathrm{H}$, $(H / 2), 7.61(\mathrm{~s}, 2 \mathrm{H}, A r I), 7.64(\mathrm{~S}, 1 \mathrm{H}, A r I I)$.

Hemicareerand $2\left(a \mathrm{CHCl}_{3}\right.$. A mixture of diol $\mathbf{1}(100 \mathrm{mg}$, $0.04 \mathrm{mmol})$ and $\mathrm{Cs}_{2} \mathrm{CO}_{3}(60 \mathrm{mg}, 0.12 \mathrm{mmol})$ in $20 \mathrm{~mL}$ of degassed NMP. The reaction mixture was stirred at $60^{\circ} \mathrm{C}$, and 3,5-bis(bromomethyl)-benzonitrile were added $(0.67$ mg, 0.12 mmol) and stirred for $36 \mathrm{hr} .3 \mathrm{~N} \mathrm{HCl}$ was added and the mixture was extracted with $\mathrm{CH}_{2} \mathrm{Cl}_{2}$. The organic layer was washed with water and brine, and then dried over $\mathrm{MgSO}_{4}$. The product was purified by silica gel chromatography with a mixture of Hexane : $\mathrm{CHCl}_{3}(2: 1)$ as a mobile phase, and recrystallized by $\mathrm{CH}_{3} \mathrm{OH}$ to give $2(40 \mathrm{mg}, 38 \%)$;
$\mathrm{FAB}+$ MASS (NOBA) m/e $2298 \quad\left(2 \cdot \mathrm{Na}^{-}, 25\right), 2397$ $\left(2(a) \mathrm{CHCl}_{3}+\mathrm{H}^{+}, 100\right) ;{ }^{1} \mathrm{H}$ NMR $\left(400 \mathrm{MH} \%, \mathrm{CDCl}_{3}\right) \delta$ 6.76-6.82 (m, 11H, $A r I I), 5.64-5.83(2 \mathrm{~d}, 8 \mathrm{H}$, outer $O C / I, \mathrm{O})$, $4.69(\mathrm{~m}, 8 \mathrm{H}$, methine), $4.16(\mathrm{~m}, 8 \mathrm{H}$, inner $\mathrm{OCH}, \mathrm{O}), 3.90(\mathrm{~d}$, $\left.12 \mathrm{H}_{4} \mathrm{OC} H_{2}\right), 3.81(\mathrm{~m}, 12 \mathrm{H}, \mathrm{CH})_{2}, 2.17(\mathrm{~s}, 8 \mathrm{H}, \mathrm{CHCH})$, $1.90\left(\mathrm{~d}, 18 \mathrm{H}, C H_{3}\right), 1.25-1.37\left(\mathrm{~m}, 40 \mathrm{H},\left(\mathrm{C} / H_{2}\right)_{5}\right), 0.90(\mathrm{l}$, $\left.12 \mathrm{H}, \mathrm{CI} I_{3}\right)$.

General procedure for the guest complexation. All complexations-decomplexalions were carried out in an argon atmosphere. The guests were used as the solvent for complexation experiments. Table 1 record the structures and the labeis of the guests, reaction tine and temperature, the isclated yield, and the pereent complexed. In each complexation, $10 \mathrm{mg}$ of host dissolved in the following specified molar excesses of guest were healed to the specified time and temperature. To the reaction mixture was added $40 \mathrm{~mL}$ of $\mathrm{CH}_{3} \mathrm{OH}$, and the precipitate was filtered, washed, and dried, and its ' $\mathrm{H}$ NMR spectrum in $\mathrm{CTOCl} 3$ taken at $25^{\circ} \mathrm{C}$. In a separate experiment, the 'H NMR of the pure guest was taken under the same conditions.

Hemicarcerplex 2a. 1.3 g (7.93 mol of 1,2,3-trimethoxybenzene, 2 a was obtained in $86 \%$ of isolation yield $(8.6$ mg). The complex guest 'H NMR $\delta-0.56\left(\mathrm{~s}, 6 \mathrm{H}, O C I_{3}\right)$, -0.46 (s, 3H, OCI $\left.I_{3}\right)$.

Hemicarcerplex 2b. $1.0 \mathrm{~g}$ (5.95 munol) of 1,3,5-trimethoxybenzene, $2 \mathrm{~b}$ was obtained in $66 \%$ isolation yield $(5.6$ Ing). The complex guest ' $\mathrm{H}$ NMR $\delta-3.28\left(\mathrm{t}, 9 \mathrm{H}, O C I_{3}\right)$.

Hemicarcerplex $2 c .1 .0 \mathrm{~mL}(8.15 \mathrm{mmol})$ of $o$-xylene. $2 \mathrm{c}$ was obtained in quantitative isolation yield. The complex guest 'H NMR $\delta-1.74\left(\mathrm{~s}, 6 \mathrm{H}, \mathrm{C} H_{3}\right)$.

Hemicarcerplex 2d. $1.0 \mathrm{~mL}(8.15 \mathrm{mmol})$ of $m$-xylene. $2 d$ was obtained in guantitative isolation yield. The complex guest 'H NMR $\delta 5.92(\mathrm{~s}, 3 \mathrm{H}, A r H),-2.00\left(\mathrm{~s}, 6 \mathrm{H}, C H_{i}\right)$.

Hemicarcerplex 2e. $1.0 \mathrm{~mL}(8.15 \mathrm{mmol})$ of $p$-xylene. $2 \mathrm{e}$ was obtained in quantitative isolation yield. The complex guest ' $H$ NMR $\delta 5.92(\mathrm{~s}, 3 \mathrm{H}, A H I),-1.99\left(\mathrm{~s}, 6 \mathrm{H}, C H_{i}\right)$.

Hemicarcerplex 2f. $1.0 \mathrm{~mL}(7.25 \mathrm{mmol})$ of 1,3-dimethoxybenzene. $2 \mathrm{f}$ was obtained in $76 \%$ of isolation yield $(2.6$ ing). The complex guest 'H NMR $\delta 4.80(\mathrm{~m}, 4 \mathrm{H}, A r I)$, $-0.60\left(\mathrm{~s}, 6 \mathrm{H}, \mathrm{OC} \mathrm{H}_{3}\right)$.

Hemicarcerplex $2 \mathrm{~g} .1 .0 \mathrm{~mL}(7.25 \mathrm{mmol})$ of 1,4 -dimethoxybenzene. $2 \mathrm{~g}$ was obtained in $65 \%$ of isolation yield $(5.5$ mg). The complex guest 'H NMR $\delta 5.30(\mathrm{~m}, 4 \mathrm{H}, A r I I)$, $-0.46\left(\mathrm{~s}, 6 \mathrm{H}, \mathrm{OC} / H_{3}\right)$. 
Acknowledgments. This work was supported by Korea Research Foundation Grant funded by the Korean Government (MOEHRD) (KRF-2003-005-C00004 and KRF-2005$005-\mathrm{J} 01102)$.

\section{References}

1. (a) Cram. D. I.: Cran. I. M. Container Holecules and Their Guests, Monographs in Stpromolecular Chemism; Stoddart. I. $F_{n}$ Ed.; The Royal Society of Chemistry: Cambridge, LK, 1994; vol. 4, Chap. 7. (b) Jasal, A.; Sherman, J. C. Chem. Rev: 1999, 99, 931.

2. (a) Warmuth, R.; Yoon, J. Acc. Chem. Res. 2001, 34, 95. (b) Cram, D. J.: Tanner, M. E.: Thomas, R. Angen: Chen. Int. Ed. Engl. 1991,30, 1024, (c) Cram, D. J.; Tanner, M. E.; Knobler, C. B. J. Am. Chem. Soc. 1991, 113,7717 . (d) Cram, D. J.; Blanda, M. T.; Pake, K.; Knobler, C. B. J. Am. Chem. Soc. 1992, 114,7765. (e) Helgeson, R. C.; Paek, K.; Knobler, C. B.; Maverick, E. F.; Cram, D. J. $J$. Am. Chem. Soc. 1996, $/ 18,5590$.

3. Cram, D. J.: Choi, H. J.: Bryant, T. A.: Knobler, C. B. J. An. Chem. Soc. 1992, 114, 7748.

4. (a) Pirondini, L.; Stendardo, A. G.; Geremia, S.; Campagnolo, M.; Samori, P.; Rabe, J. P.; Fokkens, R.; Dalcanale, E. Angew: Chem. Int. Ed. 2003, 42, 1384. (b) Ihın, H.; Ahn, J.-S.; Lah, M. S.; Ko, Y. H.: Paek K. Org. Left. 2004, 6, 3893.

5. (a) Heinz, T.; Rudkevich, D. M.; Rebek, J. Nature 1998, 394.764. (b) Chapınan, R. G. Olovsson, G.; Trotter, J.; Sherınan, I. C. J. Am. Chem. Soc. 1998, I20, 6252. (c) Choi, H.-J.; Park, Y. S.; Cho, C. S.; Koh, K.; Kim, S.-H.; Paek, K. Org. Lett. 2004, 6, 4431, (d) Rebek, J. Anger: Chem. Int. Ed. 2005, 44, 2068. (e) Palmer, L. C.;
Rebek, J. Org. Lett. $2005,7,787$.

6. (a) Castellano, R. K.; Nuckolls, C.; Eichhom, S. H.; Wood, M. R.; Lovinger, A. J.; Rebek J. J. Angen: Chem. Int. Ed. 1999, 38, 2603. (b) Klok, H.-A.; Jolliffe, K. A.; Schauer, C. L.; Prins, L. J.; Spatz, J. P.; Möler, M.: Timmerman, P.: Reinhoudt, D. N. J. Am. Chem. Soc. 1999, 12], 7154

7. (a) Levi, S. A.: Guatteri, P.; van Vegrel, F. C. J. M.: Vancso, G. J.: Dalcanale, E.; Reinhoudt. D. N. Angent: Chem., Int. Ed. 2001, 40, 1892. (b) Huisman, B.-H.; Rudhevich, D. M.; van Veggel, F. C. J. M.: Reinhoudt, D. N.J. Am. Chen. Soc. 1996, MS, 3523. (c) van Velzen, E. U. T.: Engbersen, J. F. J.: de Lange, P. J.; Mahy, J. W. G.: Reinhoudt, D. N. J. An. Chem. Soc. 1995, 177,6853 .

8. (a) Yoon. J.; Knobler, C. B.; Maverick. E. F.; Cram, D. J. Chen. Commtm. 1997, 1303. (b) Yoon, J.; Crain, D. J. Chem. Conmmtm. 1997, 1505. (c) Yoon, J.; Sheu, C.; Houk, K. N.; Knobler, C. B.; Cram, D. J. J. Org. Chen. 1996, 61, 9323. (d) Kurdistani, \$. K.: Helgeson, R. C.: Cram, D. J. J. Ant. Chent. Soc. 1995, 117 , 1659 .

9. Yoon, J.; Cram. D. J. Chem. Commur. 1997, 2065.

10. (a) Holliday, B. J.; Mirkin, C. A. Angen: Chent. Int. Ed. 2001, 40, 2022. (b) Leinnger, S.; Olenyuk, B.; Stang. P. J. Chem, Ren: 2000, 100,853 . (c) Yoshizawa, M:: Ono, K.: Kumazawa, K.: Kato, T.; Fujita, . I. J. Am. Chem. Soc. 2005, 127 , 10800. (d) Ihm, C.: Lah, M. S.: Paek, K. Bull. Korean Chem. Soc. 2005, 26, 184. (e) Ihm, C.: Kim, J.: Paek, K. Bull. Korewn Chem. Soc. 2005, 26, 805 .

11. Robbins. T. A.; Cram. D. J. J. Am. Chem. Soc 1994. J6, I11.

12. Chapınan, R. G.; Chopra, N.; Cochien, E. D.; Sherınan, J. C. J. Ant. Chem. Soc. 1994, 116, 369.

13. Segura, J. L.: Gonez, R.: Martin, N.: Guldi, D. M. Org. Lett. $2001,3,2645$ 